Lexis XXIX.2 (2005): 151-177.

\title{
Tradiciones discursivas y cambio lingüístico ${ }^{1}$
}

\author{
Johannes Kabatek \\ Universidad de Tübingen
}

Para José Luis Rivarola

1. Desde hace algunos años, el concepto de tradiciones discursivas viene siendo empleado con frecuencia cada vez mayor en la lingüística románica y, recientemente, se aplica también en el ámbito de los estudios históricos del español de América y del portugués brasileño, dedicándose a ello, en este último caso, entre otros, un Grupo de Trabajo dentro del Proyecto Para a História do Português Brasileiro (PHPB). Como suele ser habitual, la recepción de un nuevo paradigma científico provoca cierto entusiasmo, pero también cierta confusión. Lo primero está llevando a la creación de numerosos trabajos, de orientación bastante diversa, bajo la misma etiqueta de tradiciones discursivas (TD); lo segundo ha dado a luz una serie de planteamientos que procuran establecer relaciones entre el nuevo paradigma y otros, más tradicionales, como el estudio del cambio lingüístico, la pragmática o el estudio de los géneros textuales. La pregunta implícita que deriva de estos estudios es: ¿qué es realmente ese nuevo paradigma llamado tradiciones discursivas? ¿Se trata un nuevo término para algo ya conocido o de un planteamiento realmente nuevo? ¿Es sinónimo este concepto de género textual? ¿¿Se trata de formas textuales? ¿Pueden las TD también ser variedades de lenguas o estilos?

' Para una primera versión de este artículo (en versión portuguesa) véase J.K., "Tradições discursivas e mudança lingüistica", en: Tânia Lobo (ed.): Para a Historia do Português Brasileiro V, Salvador: EDUFBA, en prensa. Agradezco a Rosa María Estrada la preparación de la traducción y a Mónica Castillo sus valiosos comentarios. 
A continuación, intentaré dar algunas respuestas a este respecto, ofreciendo, en primer lugar, una precisión terminológica de lo que puede entenderse por TD y una explicación de algunos de los conceptos fundamentales ligados a ellas, relacionando, en segundo lugar, las TD con la cuestión general del cambio lingüístico, partiendo de la hipótesis de que el estudio de las TD no sólo es relevante para el estudio histórico de las lenguas sino que incluso puede ser considerado fundamental.

2. El concepto de TD nace en el seno de la lingüística alemana, especialmente dentro de la lingüística románica. Fuertemente influidos por la tradición de la escuela de Eugenio Coseriu, la mayoría de los romanistas alemanes acepta como fundamental la distinción coseriana de tres niveles del hablar, tres aspectos de la actividad lingüística cuya diferencia es considerada como requisito previo imprescindible para cualquier cuestión del estudio del lenguaje. Se trata de la distinción entre el nivel universal del hablar en general, es decir, el nivel que es común a todos los seres humanos y anterior a la diferenciación babélica de las lenguas y en el que se encuentran las características humanas universales del hablar, de la comunicación por medio de signos lingüísticos que designan el mundo de la experiencia. El segundo nivel es el histórico, el de las lenguas como sistemas de significación históricamente dados, actualizados, en el tercer nivel, en textos o en discursos concretos. Corresponde al primer nivel la designación como actividad lingüística universal, atribución de signos a un mundo de objetos, realizada desde el segundo nivel con signos de una lengua particular que, en realidad, crean una visión particular de este mundo, a partir de significados de una lengua y concretamente en una actualización en actos individuales con finalidad y sentido particulares. Los tres niveles están presentes de manera simultánea cuando se habla y únicamente se pueden derivar a partir de actos concretos, ya que no se puede hablar "universalmente" sin hablar una lengua y sin producir textos, y no se puede hablar una lengua como sistema de signos sino mediante textos. Existe, sin embargo, la posibilidad e incluso la necesidad de separar estos niveles cuando se investiga un problema lingüístico concreto.

El concepto de TD parte de esta clasificación, ampliándola y precisando algunos aspectos. Fue sobre todo a lo largo de la década de 1980 cuando se empezó a hacer hincapié, desde diferentes perspecti- 
vas, en la importancia que tiene para la lingüística la distinción entre lenguaje oral y lenguaje escrito. El origen de esos estudios se halla en la lingüística de texto y en la pragmática, tal como fueron concebidas desde las décadas de 1960 y 1970 . Ya en 1955, Eugenio Coseriu había postulado una lingúística propia del nivel del texto/discurso. ${ }^{2}$ A lo largo de la década de 1960, sobre todo en la germanística (y en gran parte de los estudios, independientemente de los postulados de Coseriu), se comenzó a estudiar el texto con sus particularidades, prestando especial atención a los tipos textuales ("Textsorten"), definidos por Peter Hartmann, en 1964, como "conjuntos de textos que comparten determinadas características." ${ }^{3}$ Una auténtica lingüistica del texto que estudia las diferentes características de tipos textuales nace como disciplina propia a inicio de la década de $1970,{ }^{4}$ y se publican diferentes trabajos que identifican las particularidades de los tipos textuales desde diversas perspectivas. En los años siguientes, se identifican diferentes campos en el estudio de la textualidad: ${ }^{5}$

- en primer lugar, se considera la textualidad a partir de los elementos lingüísticos que aparecen en cada texto, sobre todo elementos sintácticos y léxicos (p.ej., en una previsión del tiempo aparecerán formas verbales y tipos de frases diferentes de las que aparecerán en una crónica o en un tratado filosófico);

- en segundo lugar, se describe la textualidad desde el contenido, diferenciando entre la microestrutura y la macroestructura así como modelos generales (textos descriptivos, técnicos, etc.);

- en tercer lugar, se considera el texto a partir de su inserción situacional, y hasta se llega a afirmar que un tipo de situación bien determinado define un tipo preciso de texto;

- en cuarto lugar, se considera el texto desde su función o finalidad comunicativa, derivada de la ilocución dominante.

Cada una de esas tendencias tiene sus ventajas y sus desventajas, de modo que hubo autores que propusieron modelos de varios niveles, combinando los diferentes puntos de vista y a veces procurando poner en evidencia su mutua interrelación.

\footnotetext{
${ }^{2}$ Cf. Coseriu (1955-56).

${ }^{3}$ En alemán: "Mengen von Texten mit bestimmten gemeinsamen Eigenschaften", (Hartmann 1964:23).

${ }^{4}$ Cf. p. ej. el importante libro publicado por Gülich/Raible (1972).

${ }^{5}$ Para lo siguiente, véase Heinemann (2000).
} 
Si por un lado la lingüística de texto comenzó a establecerse como disciplina propia, por el otro comenzó a haber tentativas de combinar los resultados de esa nueva rama de la lingüística con otras cuestiones, particularmente la lingüística variacional y la pragmática. En el año 1983, Brigitte Schlieben-Lange, después de sus estudios con Eugenio Coseriu en Tübingen y combinando diferentes aspectos de la sociolingüística y de la pragmática con la teoría de Coseriu, presentó la propuesta de una pragmática histórica en un libro que relacionaba la discusión sobre oralidad y "escrituralidad"6 con una visión histórica y sentó las bases para lo que posteriormente se llamaría el estudio de las TD. ${ }^{7}$ En ese libro se encuentran algunas de las ideas que más tarde serían fundamentales para el concepto de las TD, entre otras la observación de que existe una historia de los textos independientemente de la historia de las lenguas y que el estudio histórico de las lenguas la debe tener en cuenta. En los años siguientes, a esas tradiciones más allá de las lenguas se les dio el nombre de tradiciones discursivas, ${ }^{8}$ y desde entonces, el concepto fue aplicado a numerosas cuestiones de diversas lenguas y se encuentra, actualmente, en el centro de interés de una serie de proyectos de investigación en el mundo entero. En los trabajos de Peter Koch y Wulf Oesterreicher, en particular en los trabajos fundamentales de Koch (1997) y Oesterreicher (1997), se define el concepto de las TD partiendo de la reduplicación del nivel histórico coseriano: se postula la existencia de dos factores a nivel histórico, la lengua como sistema gramatical y léxico de una lengua, y las tradiciones discursivas. Dicho de otra manera, se puede decir que la actividad de hablar, con una finalidad comunicativa concreta, atravesaría por dos filtros concomitantes hasta llegar al producto del acto comunicativo o enunciado: un primer filtro correspondiente a la lengua y un segundo correspondiente a las tradiciones discursivas, según el siguiente esquema: ${ }^{9}$

${ }^{6}$ Este neologismo terminológico es la traducción del alemán "Schriftlichkeit", término marcado para distinguir la escritura (medio de expresión) de la escrituralidad (concepción), es decir, del "lenguaje de distancia", cf. Koch/Oesterreicher (1985).

${ }^{7}$ Cf. Schlieben-Lange (1983). Algunas de las ideas fundamentales de este libro se pueden encontrar en versión portuguesa en Schlieben-Lange (1993).

${ }^{8}$ Cf. Koch (1997); Oesterreicher (1997).

${ }^{9}$ Cf. Oesterreicher (1997: 21); Koch (1997). 


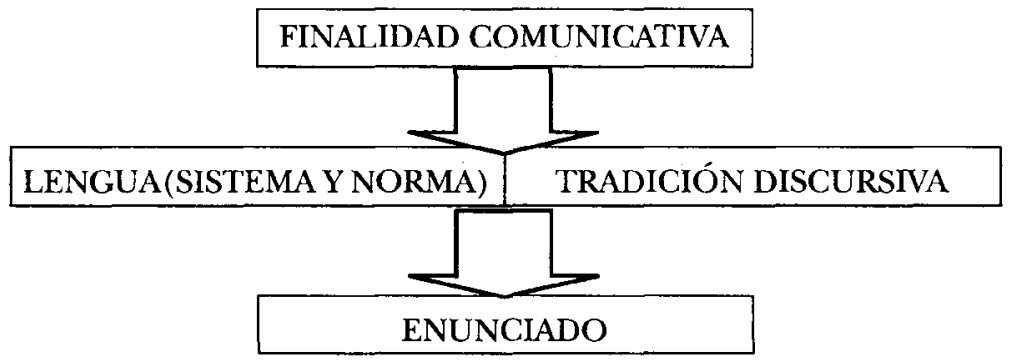

Esq. 1.: Tradiciones discursivas

Se necesita, entonces, una ampliación del concepto de historicidad, considerando que las TD compartirían la misma historicidad que las lenguas. En otro lugar expliqué por qué me parece necesario distinguir la historicidad particular de lengua, que coincide con la historicidad del ser del hombre, de la historicidad de las TD, que es de otra índole. ${ }^{10}$ Pero aunque así fuera e independientemente del lugar exacto que ocupen los dos filtros respecto a los tres niveles coserianos, debemos reconocer su existencia y su importancia a la hora de comunicarnos. De este modo, cuando me encuentro con alguien en la calle frente a mi casa por la mañana y mi intención o finalidad comunicativa es la de expresar un saludo, esa finalidad no se resuelve sólo con el acervo léxico y gramatical del español, produciendo enunciados correctos como "emito un saludo para Usted" o algo semejante, sino que digo "buenos días" según una tradición establecida más allá de las reglas de la lengua - a veces hasta contraria a las reglas actuales de la misma (según las cuales probablemente una colocación adjetiva como "día bueno" como expresión elíptica de "le deseo un buen día" sería menos marcada). Más allá de los actos de habla fundamentales como el saludo, el agradecimiento o la promesa, las tradiciones discursivas pueden estar también ligadas a finalidades más complejas exclusivas de determinadas culturas, como p. ej. las TD escritas, limitadas a las culturas con escritura, o las TD ligadas a deter-

${ }^{10}$ Véase Kabatek (2005b). Las diferentes historicidades se pueden ilustrar también con la importancia de la palabra en el cristianismo: por un lado, el hombre se define mediante la palabra, inicio de su ser y relación con la divindad (Juan 1, 1.); por el otro, existe la TD de las sagradas escrituras. 
minadas instituciones, como p. ej. los géneros jurídicos. Una primera discusión podría entender entonces las TD como formas tradicionales de decir las cosas, formas que pueden ir desde una fórmula simple hasta un género o una forma literaria compleja. Ahora, precisamente por esa relación entre las TD y los géneros, se entienden, en algunos trabajos, como sinónima las nociones de TD y de género. Pero si fuese así, el propio término TD no sería más que un sustituto para algo ya estudiado exhaustivamente por la lingüística de texto.

3. Me parece importante ampliar en dos sentidos el concepto de TD para que realmente sea útil y en cierta forma nuevo. La primera ampliación se refiere a que no queremos restringir el uso del término a tradiciones complejas (los géneros) sino aplicarlo a todos los tipos de tradiciones de textos. Una fórmula como "buenos días" no corresponde a ningún género concreto, excepto extendiendo - lo que evidentemente es posible y también ha sido propuesto más de una vez - el concepto de género también a las fórmulas tradicionales de la lengua oral. Además, proponemos una segunda ampliación, aún más importante, que podemos ilustrar con un ejemplo: como en todos los parlamentos, en la Asamblea Nacional de Francia los diferentes políticos se identifican mediante sus discursos con distintas ideas políticas, y también muestran, a través del contenido de lo expresado, su diversa procedencia. Hay, evidentemente, una identificación con contenidos políticos expresada en las proposiciones hechas respectivamente; pero se observa también otra relación de tradición cuando aquellos políticos que frecuentaron la Escuela Nacional de Administración (ENA), prestigiosa fábrica de élites, evocan su pasado usando determinadas expresiones o incluso formas gramaticales propias, como el imperfecto de subjuntivo, forma arcaica prácticamente muerta en el francés oral actual. Lo que hacen esos políticos (y pueden ser de partidos diversos) es evocar un discurso que sirve de identificador con un grupo (en este caso con elementos de una variedad diafásica concreta). Es una tradición de hablar dentro de un mismo género, el discurso parlamentario. Lo mismo ocurre también con otros géneros, y muchas veces precisamente la diferenciación interna de los géneros se hace empleando elementos de diferentes variedades lingüísticas. La existencia de diferentes tradiciones dentro de un género no niega el hecho de que el género sea también tradicional: los géneros son tradiciones de hablar, tradiciones discursivas, pero no todas las tradiciones de hablar son géneros. 
El rasgo que define las TD es, entonces, la relación de un texto en un momento determinado de la historia con otro texto anterior: una relación temporal a través de la repetición de algo. Ese "algo" puede ser la repetición total del texto entero, como en el caso de la fórmula "buenos días", pero también puede ser apenas la repetición parcial o incluso la ausencia total de repetición concreta y únicamente la repetición de una forma textual, como, por ejemplo, en el caso de dos sonetos, ligados por una tradición formal aun cuando no contengan ningún elemento concreto en común.

Tenemos ahora una serie de elementos que nos aproximan a una definición, pero todavía no son suficientes. Si por un lado una TD implica siempre la repetición de algo en el tiempo, lo contrario no es cierto: evidentemente, no todas las repeticiones de algo son tradiciones discursivas. Debemos, pues, precisar todavía una serie de condiciones. La primera es que una TD debe ser discursiva, es decir, que están excluidas todas las repeticiones no lingūísticas. Dos árboles del mismo tipo están genéticamente relacionados y pueden evocar una tradición, pero no se trata de una TD. Los anocheceres pueden considerarse "tradiciones" de la naturaleza (o de una percepción de la naturaleza) ya que se repiten, pero no son tradiciones discursivas. Y también los objetos no lingúísticos de la cultura como las pinturas cubistas, el tango o los monasterios románicos no son TD a pesar de su indiscutible tradicionalidad.

La segunda condición es que incluso en los casos de repetición de elementos lingüísticos, no toda repetición forma necesariamente una TD. En la frase anterior, palabras como "la", "segunda", "es", "que" etc. son palabras repetidas millones de veces en la lengua española en millones de circunstancias, pero ninguna de ellas es capaz de formar una TD por sí sola. " Puede haber tradiciones que consistan en una sola palabra como p. ej. una fórmula unimembre de saludo como "hola", pero en el caso de tradiciones más complejas, p. ej. la tradición del ensayo científico, sólo una combinación particular de una serie de elementos produce la inserción de un texto en una TD. Habrá, pues, que introducir en la definición esa "posibilidad inherente" de repetición, base para que la repetición pueda considerarse relevante para establecer una TD: no toda repetición de un elemento lingüístico forma una TD, pero la formación de una TD exige la re-

"Cf. también Heinemann (2000: 11). 
petición de algo. La tercera condición es la más compleja y se refiere al contenido de un texto. Se podría decir que la repetición de la comunicación de un contenido es ya una TD, ya que es algo lingüístico y al mismo tiempo algo que se repite, o la repetición de una situación y las palabras pronunciadas en ambas situaciones, o la repetición de dos instituciones o de dos canales particulares de comunicación. Todas estas repeticiones no son todavía $\mathrm{TD}$, pero son repeticiones que pueden estar íntimamente relacionadas con las TD, ligadas mediante lo que llamamos la evocación. Considerar a las TD sólo desde su lado textual únicamente tiene en cuenta un aspecto de ellas, precisamente el aspecto que nos interesa. Pero ese aspecto no es explicable sin la contrapartida que lo evoca. El saludo, por ejemplo, es evocado por una situación concreta que se repite: el mencionado encuentro evoca otros encuentros en los cuales se pronunció la misma secuencia de palabras. Lo mismo vale para las tradiciones ligadas a las instituciones: el marco concreto de la institución evoca (o lo que es lo mismo, exige) una tradición determinada. O puede haber tradiciones evocadas por el canal o medio de comunicación, como el teléfono, el SMS, el telegrama. Así, también las repeticiones anteriormente mencionadas, tanto de fenómenos naturales como de tradiciones culturales, pueden evocar TD: los anocheceres evocan la poesía de los anocheceres, que puede corresponder a una tradición discursiva, la pintura cubista evoca los discursos sobre la pintura y el tango o bien la tradición literaria de sus letras o bien la tradición de los comentarios metamusicales referidos a él. La relación de tradición de una TD tiene entonces dos fases, la TD propiamente dicha y la constelación discursiva que la evoca, de acuerdo con el siguiente esquema, en el cual el eje horizontal representa la evocación y el eje vertical la repetición, es decir, el tiempo entre los dos textos:

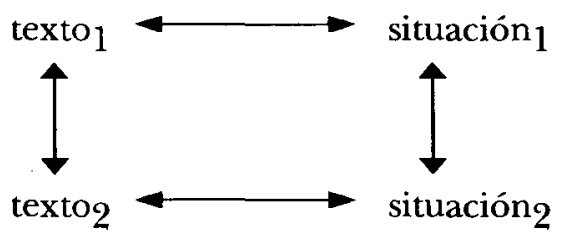

Esq. 2.: Evocación ${ }^{12}$

\footnotetext{
${ }^{12}$ Con este esquema no queremos de ningún modo defender una definición monocausal y puramente situacional de las TD; pero creemos que una relación - no necesaria, pero posible - es evidente.
} 
Este esquema evidencia además una observación: la confluencia de dos factores definidores de las TD permite completar el cuadro también en casos de ausencia de uno de los cuatro elementos. Por ejemplo, si el encuentro en la calle evoca el saludo, esta evocación funciona independientemente de si el saludo es pronunciado o no. ${ }^{1.3}$ Pero el "no saludar" tendrá entonces una función particular, y la persona no saludada se preguntará tal vez si sucedió algo entre los dos que provocó tal silencio. El silencio adquiere significado precisamente en relación con una tradición discursiva evocada, pero no enunciada. Lo mismo también es posible de manera contraria: pronunciar un saludo fuera de la situación correspondiente evoca tal situación, y se buscará el sentido de ese enunciado en relación a la situación evocada.

La TD tiene valor de signo y es reconocible por medio de signos metatextuales como "saludo", "carta", "soneto", etc.

Estamos ahora en condiciones de ofrecer una definición del concepto de TD para después precisar algunas de sus consecuencias:

Entendemos por Tradición discursiva (TD) la repetición de un texto o de una forma textual o de una manera particular de escribir o de hablar que adquiere valor de signo propio (por lo tanto es significable). Se puede formar en relación con cualquier finalidad de expresión o con cualquier elemento de contenido cuya repetición establece un lazo entre actualización y tradición, es decir, cualquier relación que se puede establecer semióticamente entre dos elementos de tradición (actos de enunciación o elementos referenciales) que evocan una determinada forma textual o determinados elementos lingüisticos empleados.

Esta definición deja todavía abierta una serie de preguntas e implica una serie de consecuencias que vamos a discutir a continuación. La primera cuestión es la relación entre tradición discursiva y referencia. En el simple ejemplo del saludo, esta relación es clara: existe una constelación referencial o situacional asociada a un texto (el saludo). Pero también existen textos no situacionales, textos independientes de una inserción pragmática concreta, p. ej. textos escritos, que crean su propia constelación discursiva. En la evolución de las

${ }^{13}$ Como ya señaló Sandig (1970: 178) "Die inhaltlich, zweckhaft und sozial bestimmte Sprachhandlungssorte bedingt die Selektion und Kombination von Sprachmitteln zu Texten." 
culturas podemos observar frecuentemente cómo las lenguas van creando textos autónomos, textos que crean sus propios entornos extralingüísticos con medios textuales, internalizando así la evocación anteriormente descrita, haciéndola inseparable de un segundo elemento: en el interior del texto, superficie textual y creación de la realidad extralingüística están fundidas.

La segunda cuestión se refiere a la relación entre tradiciones discursivas y variedades lingüísticas (variedades diatópicas, diastráticas y diafásicas). Podríamos decir simplemente que no hay una relación directa y que se trata de dos maneras diferentes de considerar el lenguaje. Pero pensando en el ejemplo de los parlamentarios franceses, también podríamos suponer que una variedad lingüística puede funcionar como tradición discursiva: la variedad diafásica que incluye el imperfecto del subjuntivo francés sería al mismo tiempo la tradición de hablar de los llamados "enarcas" que estudiaron juntos en la misma escuela. Lo que es evidente es que en los discursos de los parlamentarios hay unidad de género y no existe, dentro de esa homogeneidad, otra posibilidad de marcar diferencias que empleando elementos de diferentes variedades lingüísticas. Esto no quiere decir, sin embargo, que una TD sea lo mismo que una variedad: existen buenas razones para establecer la separación de ambos fenómenos. ${ }^{14}$ Pero el saber acerca de las variedades es transmitido a menudo mediante las TD, y el empleo situacional de elementos de variedades puede ser condicionado precisamente por las TD. ${ }^{15}$ Y el conjunto del saber que nos indica la selección de los elementos gramaticales y variacionales y las TD adecuadas es lo que puede llamarse nuestra competencia comunicativa. ${ }^{16}$

Las consecuencias de la definición son múltiples, y sólo podré mencionar algunas de ellas. La primera es que una tradición discursiva, teniendo ella misma el valor de signo, comunica más que un texto sin tradición puesto que, más allá de su valor proposicional, también transmite una referencia a una tradición concreta. "Buenos dias" no es solamente un saludo, es también una referencia a la tradición de ese

${ }^{14}$ Cf. Koch (1997: 46-49).

${ }^{15}$ En otras palabras: "Entscheidend ist in diesem Zusammenhang die schon angesprochene Tatsache, daß Diskurstraditionen keineswegs in den Regeln ciner Einzelsprache enthalten sind, daß sie aber teilweise den Einsatz bestimmter Sprachvarietäten und Verbalisierungsmuster selegieren." (Oesterreicher 1997: 20).

${ }^{16} \mathrm{Cf}$. Oesterreicher (1997: 24), en referencia a Habermas. 
saludo concreto. Por eso, una TD es más que un simple enunciado; es un acto lingüístico que relaciona un texto con una realidad, una situación, etc., pero también relaciona ese texto con otros textos de la misma tradición. Este valor adicional de una TD es el que, paradójicamente, permite su reducción. Al tener una TD valor de signo propio, puede consistir en menos de una proposición concreta y reducirse a una simple alusión a la tradición en cuestión. "Buenos días" funciona como saludo a pesar de ser una expresión elíptica. Las tradiciones de textos tienden muy frecuentemente a la elipsis y a una creciente opacidad, de manera comparable a los elementos lingüísticos que se encuentran en un canal de gramaticalización.

La segunda consecuencia de nuestra definición deriva del carácter composicional de las TD. Una TD no es siempre un texto repetido constantemente de la misma manera, puede ser también una forma textual o una combinación particular de elementos. Un texto puede, p. ej., pertencer al mismo tiempo a la TD "soneto" y "poema de amor" y contener incluso hasta más elementos tradicionales separables, p. ej. en el empleo particular del material lingüístico. Vamos a llamar a esta composicionalidad "composicionalidad paradigmática", por la confluencia de referencias a diferentes TD en un mismo fragmento del texto. Y existe también una composicionalidad "sintagmática", en la sucesión de elementos (o de subtextos) a lo largo de un texto: muchos textos no son homogéneos, contienen una serie de textos diferenciados y diferenciables. Un caso extremo es una novela como el Ulises, que juega con una sucesión de diferentes TD en el transcurso de un texto extenso.

De la composicionalidad paradigmática derivan diferentes posibilidades de transformación ${ }^{17}$ de una TD. Las TD se transforman a lo largo del tiempo, y pueden cambiar totalmente hasta convertirse en otra realidad totalmente diferente de la inicial. La variabilidad de una TD puede ser sancionada socialmente. Existen TD fuertemente fijadas, sobre todo en ámbitos religiosos o rituales o en instituciones sociales con gran valor de conservación, lugares del archivo de la memoria cultural. En otros casos, la variabilidad forma parte de la expresividad del habla, sobre todo en ámbitos orales, p. ej. en la creatividad de innovación en los chats del Internet o en otras tradiciones expuestas a las tendencias de la moda.

${ }^{17}$ Ese término ya se encuentra, con sentido semejante, en Oesterreicher (1997: 31). 
Dada la composicionalidad de las TD, la transformación de una TD puede alcanzar a uno sólo de varios aspectos concomitantes. Para dar un ejemplo, podemos observar varios casos de la épica medieval en los que un poema épico, ligado a una forma textual particular, es transformado, apareciendo insertado en textos historiográficos como parte de la narración. Tal es el caso, p. ej., del Poema de mio Cid, que aparece con posterioridad a la versión épica en toda una serie de crónicas medievales a partir del siglo XIII. La prosa historiográfica sigue otro patrón textual que el de la épica; estamos, pues, frente a una transformación de una forma textual en otra, manteniéndose el mismo contenido. Lo interesante es que en los casos de transformación, estando implícitamente presente el modelo textual original, a menudo puede observarse la presencia de elementos particulares de la forma textual inicial. ${ }^{18}$

Ampliando el concepto de Interferencia lingüistica, podemos hablar aquí de "interferencia textual", interferencia entre dos TD. Por definición, siempre que haya transformación de una TD, habrá interferencia. En trabajos anteriores sobre la interferencia lingüística, ${ }^{19}$ he observado que siempre es necesario distinguir entre dos tipos de interferencia, la interferencia positiva y la interferencia negativa. La interferencia positiva es lo que generalmente se entiende por interferencia (IF) sin más, en el caso de la IF lingüística, la presencia de elementos de una lengua A en un texto de la lengua B. La IF negativa, por el contrario, muchas veces no es considerada en los estudios de IF y consiste en la ausencia de determinados elementos en un texto de la lengua $\mathrm{B}$ debido a la presencia de la lengua $A$. Es una IF que no produce resultados que son "errores" abiertamente visibles, sino simplemente alteraciones en la frecuencia del empleo de ciertas formas. Hay dos tipos de IF negativa, la IF de convergencia y la IF de divergencia. La primera consiste - y sigo con el caso de la IF entre lenguas - en la preferencia por formas comunes a las dos lenguas, evitando formas diferentes. La segunda es lo con-

${ }^{18}$ En el caso mencionado de las versiones del Poema de mio Cid en las crónicas, se observa, p. ej., la presencia de rimas asonantes, no usuales normalmente en crónicas, como en la Crónica de Castilla: "Estonce fabló don Álvar Fáñez, su primo cormano: - Conbusco iremos todos, Cid, por yermos y por poblados, y nunca vos falleceremos en cuanto seamos bivos y sanos; conbusco despenderemos las mulas y los cavallos, y los averes y los paños; sienpre vos serviremos commo leales amigos y vasallos. - Estonce otorgaron todos lo que dixo Álvar Fáñez y mucho les agradesció mio Cid cuanto allí fue razonado."

${ }^{19}$ Véase Kabatek (2000a). El concepto está tomado de Coseriu (1977). 
trario: la preferencia por formas diferentes, evitando formas comunes. Esos dos tipos de IF negativa pueden estar presentes simultáneamente en la producción de un texto y son particularmente frecuentes en el contacto de lenguas semejantes (como p. ej. el portugués y el español). Ahora bien, el concepto de IF negativa también es aplicable en el caso de la IF entre TD. Siguiendo con el ejemplo de la épica, el autor de una crónica, consciente de la diferencia de las características textuales entre épica y crónica, puede verse tentado a evitar cualquier alusión al género original, huyendo cualquier repetición de secuencias de vocales interpretable como rima. De manera parecida, el traductor de un texto de una lengua a otra semejante puede intentar buscar expresiones diferenciadas del original incluso cuando las expresiones del original son totalmente "normales" también en la lengua de llegada. En el estudio de las transformaciones de las TD, el aspecto de la IF textual, tanto positiva como negativa, es uno de los más importantes, siendo la transformación muchas veces nada más que la combinación nueva de elementos procedentes de diferentes tradiciones.

4. El estudio de las tradiciones discursivas tiene numerosas aplicaciones. Una de ellas es la relación con la gramática histórica, para acercarnos a una gramática histórica más diferenciada que deje vislumbrar con más rigor las evoluciones de la lengua y los procesos del cambio lingüístico. Muchas veces se piensa que la evolución de una lengua es un proceso rectilíneo y que el objetivo del historiador de la lengua es la reconstrucción de esa especie de "diacronía ideal", la evolución de la lengua como si fuera una simple línea. Pero en realidad, cuando se estudia la historia de una lengua, lo que se estudia no es la evolución de la lengua (recordemos la tripartición de niveles) sino textos de diferentes épocas, textos que se consideran representativos de los respectivos estados de lengua (états de langue en la terminología de Saussure), de acuerdo con el siguiente esquema:

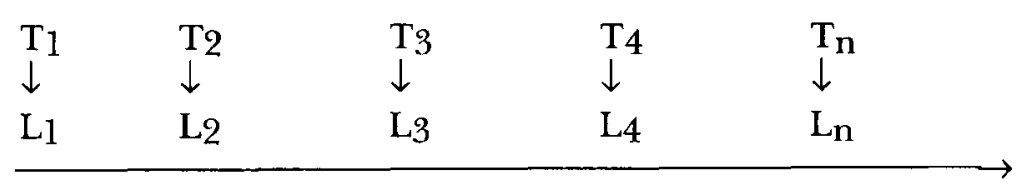

t

Esq. 3: Tradición textual e historia de la lengua 
En diversos momentos de la historia se escogen diferentes textos para la construcción de una sucesión histórica de gramáticas. Esta visión es problemática si pensamos que las diferentes tradiciones discursivas también condicionan el uso de los medios lingüísticos empleados y que puede haber variación de esos medios según la TD. Una posibilidad para evitar desviaciones provocadas por los diferentes textos es la de basar el estudio histórico únicamente en una tradición discursiva. Pero entonces no estudiamos la historia de la lengua, sino la historia de esa tradición discursiva. Otra solución es la de mezclar textos de diferentes tradiciones para trabajar con un corpus "equilibrado" de textos, lo cual, como se sabe, es una de las exigencias más importantes para el diseño de córpora "representativos". Pero ¿̨qué quiere decir realmente "equilibrado"? ¿Cuál es la mezcla más acertada para una buena historia de la lengua? Algunos lingüistas creen que se trata de un problema de cantidad. Si trabajamos con córpora extensos, el problema de la variación parece ser menor. ${ }^{20}$ Parece serlo, pero supongo que simplemente será menos visible: también los macrocórpora cada vez más disponibles para los estudios históricos de las lenguas están compuestos de textos, y no hay un equilibrio objetivo de textos en una lengua. Hay textos, textos de diferentes tradiciones discursivas, textos de una misma lengua, pero no objetivamente cuantificables. Una lengua no es como una receta de cocina con ingredientes textuales, que pueden medirse en gramos y litros. La única solución al problema de la coexistencia de tradiciones discursivas diferentes que influyen sobre la diacronía de la lengua es entonces una historia de la lengua que estudie las diferentes tradiciones sin limitarse a una solamente, manteniendo la diferenciación, es decir, una historia de la lengua menos monolítica que permitirá saber en qué TD se crea una innovación, cómo se difunde a través de las TD, y también dónde hay TD resistentes a las innovaciones, TD que preservan elementos que en otras TD ya no se usan.

Una vez que se acepta esa propuesta, habrá que solucionar el problema metodológico de cómo se puede estudiar la historia de una lengua diferenciando las TD. Una posibilidad es el estudio filológico detallado de los textos: podemos explicar cada fenómeno gramatical en cada texto con referencia a la TD de ese texto. Pero el problema

\footnotetext{
${ }^{20}$ Véanse algunas de las discusiones en Pusch/Kabatek/Raible (2005).
} 
del estudio detallado, ilimitado en principio en su descripción de elementos, es que complica la comparación y la perspectiva de conjunto. Entre los dos extremos -descripción de una evolución general de la lengua sin diferenciación de detalles textuales y descripción de detalles textuales perdiendo de vista la evolución de la lengua- tiene que haber un camino intermedio. Una propuesta importante en ese sentido es la metodología llamada "Análisis multidimensional" (Multidimensional analysis) aplicada por Douglas Biber en el estudio de cónpora históricos del inglés y de otras lenguas. Biber parte de la hipótesis de que lo que permite la identificación de una $\mathrm{TD}^{21}$ es una particular combinación de elementos en un texto. En un corpus histórico de la lengua inglesa, Biber analiza 67 elementos de 16 clases tan diversas como marcadores de tiempo y aspecto, adverbios de tiempo y lugar, pronombres, preguntas, formas nominalizadas, subordinación, preposiciones, clases léxicas, clases verbales, etc. El primer resultado de los datos encontrados se somete a un análisis factorial para determinar cuáles de los elementos pueden correlacionarse. Después, se mide la evolución de los diferentes factores a lo largo de la historia y en relación a las diferentes $T D$.

Una segunda vía, semejante a la de Biber, es la que estamos siguiendo en un proyecto en la Universidad de Tübingen. La diferencia fundamental con respecto al método de Biber es que no partimos de un análisis "ciego" de elementos sino de un esquema cognitivo y sintáctico que reduce la cantidad de elementos por medir, los cuales están ordenados de una forma que permite la máxima diferenciación en la interpretación con un esfuerzo bastante reducido. Partimos de una teoría propuesta hace algunos años por el lingüista alemán Wolfgang Raible ${ }^{22}$ y cuyo propósito consiste en el análisis de los elementos que relacionan los componentes proposicionales en un texto. Raible llama "Junktion", junción (término adoptado de Lucien Tesnière) una dimensión universal del lenguaje según la cual pueden sistematizarse los diferentes elementos y las diferentes técnicas linguísticas para

${ }^{21}$ Biber no habla de TD, sino de registros o de tipos de textos, refiriéndose en parte a una clasificación externa de los textos. No podemos discutir aquí el problema de la categorización externa en relación a la objetividad de las características de los textos, pero queremos señalar que las tradiciones de textos identificadas por Biber corresponden en gran medida a lo que llamamos TD.

22 Para más detalles remito a Raible (1992). Véase también Raible (2001). 
juntar o combinar elementos: técnicas, por un lado, clasificadas según un esquema sintáctico que describe diferentes grados de lo que Raible llama "integración” (desde la simple yuxtaposición hasta las formas extremas como la integración por nominalización, pasando por diferentes etapas de coordinación y de subordinación), y clasificadas, por otro lado, según las relaciones semánticas expresadas por los elementos de junción, los cuales siguen una escala cognitiva de complejidad creciente (comenzando con relaciones poco complejas como la condicionalidad hasta relaciones más complejas como causalidad, finalidad, concesividad), según el siguiente esquema:

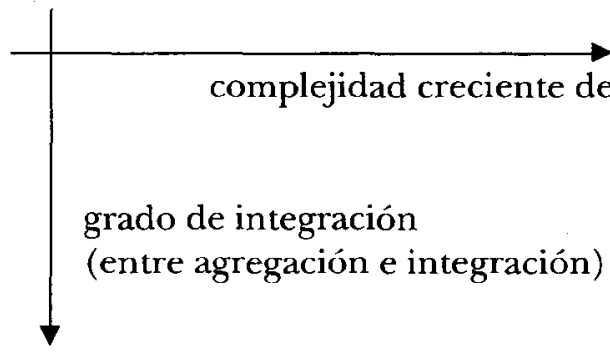

Esq. 4: "Junción" según W. Raible

Hablar consiste fundamentalmente en la atribución de signos lingüísticos a un mundo percibido, signos con carácter nominal (para designar cosas) o verbal (para designar acciones). Entre las acciones designadas con la combinación de elementos nominales y verbales, es decir, entre frases, los hablantes y los oyentes establecen relaciones de diferentes tipos, clasificables según su grado de complejidad. Estas relaciones pueden estar implícitas o pueden tener representación en la superficie textual por medio de juntores: elementos deícticos, elementos de coordinación o de subordinación (conjunciones), construcciones absolutas, construcciones de participo, grupos preposicionales, preposiciones simples, etc. El esquema de junción ofrece un orden lógico a esos elementos. El orden del grado de integración de los juntores en el eje vertical se puede ilustrar mediante los siguientes ejemplos, en este caso de causalidad, aunque es imaginable, en principio, cualquier relación semántica en cualquier nivel entre agregación e integración: 
I. Yuxtaposición sin junción (Juan está enfermo. No come nada.) II. Relación deíctica con la frase anterior ( [...] Por eso, no come nada.)

III. Oraciones explícitamente unidas ( $[\ldots]$ pues está enfermo.)

IV. Subordinación (Juan no come nada porque está enfermo.)

V. Construcciones gerundiales o participiales (Estando enfermo, Juan no come nada.)

VI. Grupos preposicionales (Por causa de enfermedad, Juan no come nada.)

VII. Preposiciones (Por enfermedad, Juan no come nada.)

Esq. 5: Esquema simplificado del eje vertical de junción (grado de integración)

Así como los juntores están insertados en el eje vertical, también lo están en el eje horizontal, de acuerdo con el tipo semántico del nexo que expresan: en el nivel vertical de la subordinación (o de las conjunciones subordinantes), podemos distinguir juntores como $s i$ para la condicionalidad, porque para la causalidad, para que para la finalidad, aunque o no obstante que, etc. para la concesividad, etc. El objetivo del esquema de junción es ofrecer una clasificación de los juntores en una lengua en la que cada juntor se localiza mediante dos coordenadas, p. ej. un juntor como para que está localizado en el eje vertical en el nivel IV, de subordinación, y en el eje horizontal 8, de finalidad:

\begin{tabular}{|l|l|l|l|l|l|l|l|l|l|}
\hline & 1 & 2 & 3 & 4 & 5 & 6 & 7 & 8 & 9 \\
\hline II & & & & & & & & & \\
\hline II & & & & & & & & & \\
\hline IV & & & & & & & & $\begin{array}{c}\text { para } \\
\text { que }\end{array}$ & \\
\hline VI & & & & & & & & & \\
\hline VII & & & & & & & & \\
\hline
\end{tabular}

Esq. 6: Localización de un juntor con dos coordenadas 
Raible establece toda una serie de correlaciones de los parámetros de junción con otros factores como la evolución de las lenguas, la adquisición del lenguaje, la gramaticalización y la relación entre oralidad y escrituralidad. Si el objetivo del trabajo de Raible fue, en principio, tipológico, queriendo ofrecer un instrumento para la comparación de las lenguas (¿cuáles son los juntores existentes en una lengua?, ¿cuál es su respectivo origen?, ¿existen técnicas particulares en una lengua que no se encuentran en otra?, ¿cuáles son las tendencias universales de evolución?, etc.), nuestra aplicación del esquema es textual, partiendo de dos observaciones: primero, que el empleo de los juntores, también en una misma sincronía, varía según el texto que se escriba; y segundo, que esa variación, si se considera que se observó también una evolución diacrónica de los juntores, será probablemente el reflejo sincrónico de esa evolución diacrónica.

En nuestro proyecto, en una serie de trabajos previos hemos procurado determinar la relación entre los juntores que se encuentran en un texto y la TD a la cual pertenece el texto, pudiendo afirmar, por lo menos según los primeros estudios, que existe una clara correlación. Esta correlación es por un lado cualitativa, lo cual quiere decir que en una TD de finalidad determinada va a aparecer una serie de nexos que corresponden al contenido expresado en ese texto. Pero la posibilidad de distinguir diferentes TD a través del empleo de juntores es todavía mucho mayor cuando se introduce un elemento de cantidad relativa, contando el número relativo de juntores que aparecen en un texto. Los dos factores nos conducen a la siguiente hipótesis de trabajo:

Los esquemas de junción de un texto - juntores que contiene y frecuencia relativa - son síntomas para determinar la tradición discursiva a la que el texto pertenece.

El punto de partida de nuestros análisis fue un trabajo filológico extenso sobre diferentes TD del siglo XIII castellano. ${ }^{23}$ En muchos estudios de gramática histórica, es usual introducir diferenciaciones textuales, del tipo: "lengua escrita / hablada"; "textos literarios / no literarios”, “textos científicos”, “poéticos”, “jurídicos”, etc. Tales diferenciaciones nos parecen totalmente insuficientes ya que mostramos en los estudios previos cómo textos supuestamente del mismo ámbito - en nuestro caso textos jurídicos - pueden estar bien diferenciados y

${ }^{23}$ Sobre el particular remito a Kabatek (2005). 
pertenecer a TD bastante diversas. Identificamos por lo menos tres mundos jurídicos diferentes en la Edad Media castellana, el mundo del derecho oral transmitido por escrito en fazañas o noticias de casos jurídicos concretos; el mundo del derecho foral (los fueros) en la tradición de la Península Ibérica desde la época visigoda y, finalmente, un tercer mundo que corresponde al renacimiento del derecho romano, divulgado desde Italia, sobre todo a partir de la segunda mitad del siglo XII. Se trata de tres mundos con tres tradiciones de textos, tradiciones temporalmente sucesivas pero también concomitantes dada la supervivencia de las dos primeras a lo largo del siglo XIII, época de la plena recepción del derecho romano en la península Ibérica. Dentro de un mismo "género" (el jurídico) y para una misma finalidad expresiva ("texto jurídico normativo") encontramos, pues, tres TD diferentes, identificadas mediante un análisis que aprovecha, entre otras, las informaciones secundarias ofrecidas por los historiadores del derecho.

Siguiendo nuestra hipótesis, esas tres TD deberian presentar diferencias en la superficie textual, diferencias identificables gracias al análisis multidimensional o mediante un análisis de los esquemas de junción.

Escogimos tres textos "prototípicos"24 de esas TD para el análisis de junción. Éste se realiza a través de tres etapas semi-automatizadas y apoyadas en componentes de un programa computacional elaborado dentro de nuestro proyecto. El primer paso consiste en la identificación semiautomática de las conjunciones en los diferentes textos. El segundo paso - tratándose de textos medievales - en la unificación grafemática. Finalmente, se procede a un cálculo cuantitativo: se mide la cantidad absoluta de juntores contenida en cada fragmento de 1000 palabras, sumando los resultados de cada fragmento para establecer la cantidad relativa de los juntores por 1000 palabras. Los resultados del estudio piloto son los siguientes: ${ }^{25}$

${ }^{24}$ La identifícación histórico-filológica de las TD muestra que también hay textos donde las tres TD se entrecruzan, textos menos prototipicos, productos ya de transformaciones de las TD.

${ }^{25}$ Los textos de nuestros análisis están publicados en las páginas www.kabatek.de/bologna y www.kabatek.de/codi El primer texto es la colección de Fazañas conocida como Fazañas de Palenzuela, el segundo texto una colección de fueros contenida en el Libro de los fueros de Castiella, y el tercero la Suma de derecho romano llamada Lo codi en su versión castellana, cuya versión original provenzal, que data de la segunda mitad del siglo XII, es el primer texto extenso de derecho romano en lengua románica. 


\begin{tabular}{|c|c|c|c|c|c|c|c|c|c|}
\hline (1) & 1 & 2 & 3 & 4 & 5 & 6 & 7 & 8 & 9 \\
\hline 11 & & & & & 1 & & & & \\
\hline III & 114 & & & & & & & & \\
\hline$\overline{1 Y}$ & & & & & & & & 1 & \\
\hline$V$ & & & & & & & & & \\
\hline$\sqrt{11}$ & & & & & & & & & \\
\hline VII & & & & & & 2 & & & \\
\hline
\end{tabular}

Esq. 7: Esquema de junción en las Fazañas de Palenzuela

El primer texto, una colección de "Fazañas", es un texto "simple" que describe hechos, casos jurídicos concretos mediante la enumeración de acontecimientos. Los elementos de junción son mínimos; se trata casi únicamente de frases cortas de nombre y verbo unidas con la conjunción et, presente con alta frecuencia. Las pocas "excepciones” en el texto se deben a la presencia de fórmulas latinizantes.

El segundo texto, un texto foral, corresponde a una lista de frases que sigue un modelo prototípico de "ley" conocido desde la antigüedad. Es, fundamentalmente, una sucesión de frases condicionales: si $a$ hiciera $b$, será sancionado con la sanción $c$ :

\begin{tabular}{|c|c|c|c|c|c|c|c|c|c|}
\hline (5) & 1 & 2 & 3 & 4 & 5 & 6 & 7 & 8 & 9 \\
\hline$\pi$ & & & & & & & & & \\
\hline III & 29 & & & & 1 & & & & 1 \\
\hline TV & & & 26 & & & 1 & & & \\
\hline$V^{\prime}$ & & & 3 & 1 & & & & & \\
\hline $\mathbf{M}$ & & & & & & & & & \\
\hline VII & & & & 12 & & & & & \\
\hline
\end{tabular}

Esq. 8: Esquema de junción en un fragmento del Libro de los fueros de Castiella

El tercer texto es resultado de la recepción del derecho romano re-descubierto en la Edad Media. Ese "nuevo" derecho pretende unificar la diversidad de las leyes, introduciendo un sistema jurídico universal, aplicable a todos los casos posibles; un derecho caracterizado por la discusión científica de las materias jurídicas con una organiza- 
ción completa y una gran cantidad de normas metajurídicas. Los textos correspondientes están bastante elaborados y presentan un amplio espectro de posibilidades de junción:

\begin{tabular}{|c|c|c|c|c|c|c|c|c|c|}
\hline II & 1 & 2 & 3 & 4 & 5 & 6 & 7 & 8 & 9 \\
\hline II & & & & & & 1 & & & 5 \\
\hline IV & & & 19 & 13 & 1 & 7 & & & 1 \\
\hline$V$ & & & & & & & & & \\
\hline $\mathrm{V}$ & & & & & & & & & \\
\hline $\mathrm{VI}$ & & & & 1 & & 4 & & 1 & \\
\hline
\end{tabular}

Esq. 9: Esquema de junción en un fragmento de Lo codi

Sintetizando el análisis de los textos, podemos visualizar el resultado de diferentes maneras. Una posibilidad es sumar los resultados de cada eje de las dos dimensiones del esquema, como en los gráficos siguientes:

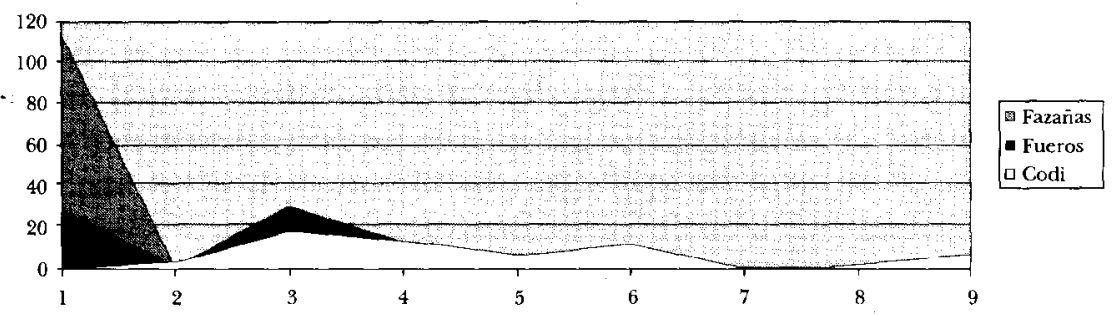

Esq. 10: Relaciones semánticas de los juntores en los tres textos

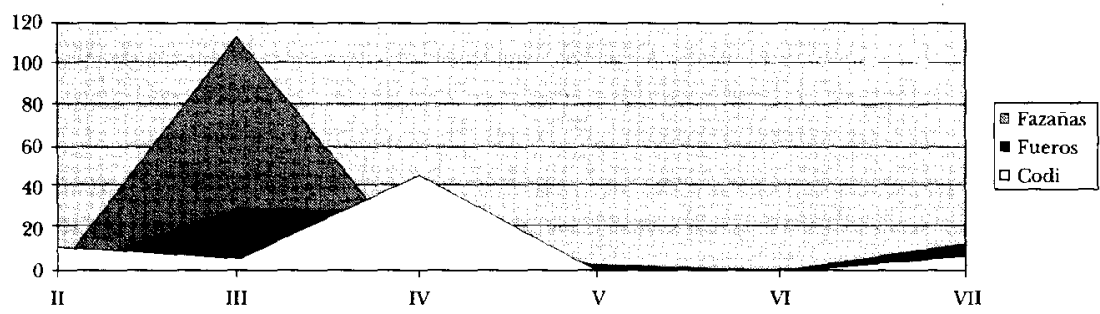

Esq. 11: Presencia relativa de grados de integración de los juntores en los tres textos 
Las diferencias son evidentes: en las Fazañas predomina, en cuanto a las rèlaciones (esq. 10), la simple adición, con frecuencia muy elevada. La relación dominante en lós Fueros es la condicionalidad, y en el texto de derecho romano, en nuestro ejemplo la traducción castellana de la Suma lo codi (en su versión original provenzal el primer texto extenso de derecho romano en lengua románica), se observa un espectro amplio de posibilidades, desde la adición hasta la concesividad, pasando por la condicionalidad, la causalidad, la consecutividad, la finalidad, etc. En cuanto a la dimensión sintáctica, de diferentes grados de integración (esq. 11), las Fazañas se limitan a frases principales sin subordinación; en los Fueros predomina la subordinación, y el texto de derecho romano presenta, más allá de la subordinación, otras técnicas de in tegración mediante grupos preposicionales, frases gerundiales, nominalizaciones, etc.

En este estudio de textos jurídicos medievales, obtuvimos una primera confirmación de que las diferencias textuales-gramaticales son indicadores, síntomas de diferentes TD. Pero nuestra hipótesis implica también la dirección inversa: los esquemas de junción que presenta un texto serán relevantes para la determinación de la TD a la que el texto pertenece.

Nuestro ejemplo parece estar alejado de la realidad actual de las lenguas vivas; sin embargo, nos paréce que los principios elaborados sobre la base del ejemplo español medieval serán aplicables, en principio, a cualquier estudio de TD en las lenguas románicas. ${ }^{26}$ Este tipo de estudio permite diferentes observaciones:

- la supuesta homogeneidad de un "género" puede presentar una heterogeneidad interna considerable, observable, al lado de otras posibilidades, identificando síntomas textuales diferentes para cada TD.

- esa heterogeneidad interna señala diferentes TD, y a la indicación de los síntomas debe seguir la interpretación histórica de los datos para llegar a describir p. ej. cuáles son las diferentes TD que confluyen en un mismo género.

5. La propuesta de partir del análisis de los esquemas de junción para tener un criterio de determinación de las TD no es evidentemente

${ }^{26}$ Y también en otras lenguas; puede ser que haya lenguas que por sus caracteristicas tipológicas no permitan un análisis de este tipo sin mayores modificaciones metodológicas, pero nos parece viable, en principio, la aplicación de la metodología por lo menos a todas las lenguas indoeuropeas. 
la única posible, pero lo importante es el principio: creemos necesaria una linguística histórica de corpus con diferenciación de TD distintas, y creemos que la vía para su establecimiento debe pasar por el intento de cuantificación de elementos en los textos que se puedan considerar síntomas textuales, síntomas para determinadas TD. En los próximos años, procuraremos mejorar la metodología esbozada y ofrecer resultados concretos de aplicación en diferentes lenguas románicas.

Este tipo de estudio de las TD es de enorme importancia para la cuestión del cambio lingüístico. Partimos de una crítica de la visión monolítica de la gramática histórica considerando que conviene diferenciar diversas TD. Esta crítica no ofrece una explicación del cambio lingüístico, pero indica algunas conexiones entre el cambio y las TD. La lengua no es una entidad monolítica que se desarrolla como tal a lo largo del tiempo. Es un conjunto de variedades y de tradiciones discursivas con evoluciones internas bien diferenciadas: una innovación se da, en general, en un texto determinado, en un texto que pertenece a una TD. A partir de ahí la innovación puede generalizarse en esa tradición, pero aún no es general en la lengua: hace falta la adopción de la innovación en otras tradiciones. Y tal como una innovación se puede localizar, teóricamente, en un texto y en una TD concreta, el proceso contrario, o sea la pérdida de elementos en una lengua, tampoco es general y repentino en toda la lengua sino que comienza en algunas TD hasta tal vez alcanzar a todas. Sin embargo, frecuentemente los elementos se encuentran fosilizados durante mucho tiempo en algunas TD antes de su desaparición total. Para la teoría del cambio, es imprescindible tener en cuenta la importancia de la relación entre TD y evolución de la lengua. Y para la historia de una lengua concreta, dos postulados metodológicos derivan de esa observación: en los estudios de evoluciones diacrónicas de elementos particulares de una lengua, por detrás de la evolución abstracta de un elemento en la sucesión del tiempo, conviene diferenciar, en lo posible, la diversidad textual real, base de la interpretación acertada de la evolución lingüística. Y para la visión de conjunto de la historia de la lengua, conviene sustituir el instrumento de investigación "corpus diacrónico" por un corpus diacrónico multidimensional, un corpus de textos pertenecientes a diferentes TD, con posibilidad de proyección diacrónica, pero también con posibilidad de una visión diferenciada de las distintas TD coexistentes a lo largo de la historia de una lengua. 
6. La innovación del concepto de TD puede parecer de poca importancia cuando es planteada dentro de la lingüística del texto y si consideramos las diferentes características de las TD vamos a encontrar pocos elementos nuevos con respecto a la gran cantidad de trabajos sobre géneros textuales publicados desde los años 70 y 80 . Lo importante de este concepto parece residir sobre todo en el retorno a otros campos de la lingüística, y, en particular, a la lingüística histórica. En los últimos años, se observan tendencias en la lingüística que proclaman la muerte de la lingüística histórica, y en ciertas escuelas y en ciertos centros parece que se trata realmente de una disciplina moribunda. ${ }^{27}$ No obstante, en estos mismos años también ha habido toda una serie de innovaciones que han abierto perspectivas nuevas, desde la visión pragmática o la visión cognitiva hasta la perspectiva propuesta desde las TD. El estudio de la historia de la lengua a partir de las TD no proporciona un paradigma diferente para sustituir otras perspectivas, al contrario, parece perfectamente compatible con otros enfoques. Lo que propone es una perspectiva más diferenciada de la lingüística histórica y tal vez en algunos casos permitirá la solución de algún caso empírico concreto todavía abierto.

Para la investigación futura, una vez aclaradas las cuestiones terminológicas y algunos de los parámetros básicos, me parece fundamental seguir estudiando la relación entre análisis cualitativo y análisis cuantitativo, para ver cuáles de los aspectos de las TD son cuantificables y cuáles no. La cuantificación de elementos nunca va a ser un sustituto del análisis filológico de detalles, pero es una base objetiva para la comparación, fundamento de cualquier estudio de evolución histórica.

${ }^{27}$ En el número especial $31-1$ de la revista La Corónica se discutió sobre la cuestión de la supuesta muerte de la lingüística histórica. Creo que es una muerte geográficamente muy limitada ya que existen importantes centros en el mundo que están contribuyendo a la continua innovación de los estudios históricos. Cf. Kabatek (2003). 


\section{BIBLIOGRAFÍA}

Aschenberg, Heidi

2003 "Diskurstraditionen - Orientierungen und Fragestellungen". En Aschenberg/Wilhelm (eds.), 1-18.

Aschenberg, Heidi/Wilhelm, Raymund (ed.)

2003 Romanische Sprachgeschichte und Diskurstraditionen. Tübingen: Narr.

Callou, Dinah et alii

2003 Análise contrastiva de variedades do português. Rio de Janeiro: In-Fólio.

Coseriu, Eugenio

1955-56 "Determinación y entorno. Dos problemas de una lingüística del hablar". Romanistisches Jahrbuch VII, 29-54.

1977 "Sprachliche Interferenz bei Hochgebildeten". En Herbert Kolb / Hartmut Lauffer (eds.). Sprachliche Interferenz: Festschrift für Werner. Tübingen: Niemeyer, $77-100$.

1980 Textlinguistik. Eine Einführung, 3a ed. revisada. Tübingen: Francke. 1994.

Gülich, Elisabeth / Raible, Wolfgang (eds.)

1972 Textsorten. Differenzierungskriterien aus linguistischer Sicht. Frankfurt

Hartmann, Peter

1964 “Text, Texte, Klassen von Texten". Bogawus 2, 15-25.

Heinemann, Peter

2000 "Textsorten. Zur Diskussion um Basisklassen des Kommunizierens. Rückschau und Ausblick". En Kirsten Adamzik (ed.). Textsorten. Reflexionen und Analysen. Tübingen: Stauffenburg, 9-29.

Jacob, Daniel/Kabatek, Johannes (eds.)

2001 Lengua medieval y tradiciones discursivas en la Peninsula Ibérica: descripción gramatical - pragmática histórica - metodologia. Frankfurt/Madrid: Vervuert/Iberoamericana (Lingüística Iberoamericana).

Kabatek, Johannes

2005a Die Bolognesische Renaissance und der Ausbau romanischer Sprachen - Juristische Texttraditionen und Sprachentwicklung in Südfrankreich und Spanien im 12. und 13. Jahrhundert. Tübingen: Niemeyer (Beihefte zur Zeitschrift für Romanische Philologie 321). 
2005b "Sobre a historicidade de textos", tradução de José da Silva Simões. Linha d'água (São Paulo) 17, 159-170.

2003 "La lingüística románica histórica: tradición e innovación en una disciplina viva”. La Corónica 31.235-40.

2002 "Oralidad, proceso y estructura". Pandora (Paris) 2, 2: 37-54.

2001 “Cómo investigar las tradiciones discursivas medievales? El ejemplo de los textos jurídicos castellanos". En Jacob/Kabatek (eds.), 97132.

2000a Os falantes como lingüistas. Tradición, innovación e interferencias no galego actual. Vigo: Xerais.

$2000 \mathrm{~b}$ "L'oral et l'écrit - quelques aspects théoriques d'un « nouveau » paradigme dans le canon de la linguistique romane". En Wolfgang Dahmen/Günter Holtus/Johannes Kramer/Michael Metzeltin/Wolfgang Schweickard/Otto Winkelmann (eds.). Kanonbildung in der Romanistik und in den Nachbardisziplinen. Romanistisches Kolloquium XIV. Tübingen: Narr, 305-320.

Koch, Peter

1997 "Diskurstraditionen: zu ihrem sprachtheoretischen Status und ihrer Dynamik". En Barbara Frank/Thomas Haye/Doris Tophinke (eds.). Gattungen mittelalterlicher Schriftlichkeit. Tübingen: Narr, 43-79.

Koch, Peter y Wulf Oesterreicher

1994 "Schriftlichkeit und Sprache". En Hartmut Günther/Otto Ludwig (ed.). Schrift und Schriftlichkeit. Ein internationales Handbuch / Writing and its Use. An international Handbook, 2 vols. Berlin/New York: Mouton de Gruyter, vol. I, 587-604.

1985 "Sprache der Nāhe - Sprache der Distanz. Mündlichkeit und Schriftlichkeit im Spannungsfeld von Sprachtheorie und Sprachgeschichte $^{\text {. }}$. Romanistisches Jahrouch 36, 15-43.

Oesterreicher, Wulf

1997 "Zur Fundierung von Diskurstraditionen". En Thomas Haye/Doris Tophinke (eds.). Gattungen mittelalterlicher Schriftlichkeit. Tübingen: Narr, 19-41.

Pusch, Claus, Johannes Kabatek y Wolfgang Raible (eds.)

2005 Romanistische Korpuslinguistik II: Korpora und diachrone Sprachwissenschaft. Romance Corpus Linguistics II: Corpora and Diachronic Linguistics. Tübingen: Narr (ScriptOralia 130).

Raible, Wolfgang

2001

"Linking clauses". En Martin Haspelmath/Ekkehard König/Wulf Oesterreicher/Wolfgang Raible (eds.). Language Typology and Language 
Universals. An International Handbook, I. Berlin/New York: de Gruyter, 590-617.

1992 Junktion: eine Dimension der Sprache und ihre Realisierungsformen zwischen Aggregation und Integration. Heidelberg: Winter.

Sandig, Barbara

1993

História do falar e história da lingüística. Campinas: Unicamp.

1970

"Probleme einer linguistischen Stilistik". Linguistik und Didaktik 3, 177-194.

Schlieben-Lange, Brigitte

1983

Traditionen des Sprechens. Elemente einer pragmatischen Sprachgeschichtsschreibung. Stuttgart: Kohlhammer. 